\title{
EXPONENTIAL MARTINGALES AND CIR MODEL
}

\author{
WOJCIECH SZATZSCHNEIDER \\ School of Actuarial Sciences \\ Universidad Anahuac Mexico Norte, Mexico \\ E-mail:wojciech@anahuac.mx
}

\begin{abstract}
With the use of exponential martingales and the Girsanov theorem we show how to calculate bond prices in a large variety of square root processes. We clarify and correct several errors that abound in financial literature concerning these processes. The most important topics are linear risk premia, the Longstaff double square model, and calculations concerning correlated CIR processes.
\end{abstract}

0. Introduction. The Cox, Ingersoll \& Ross model for interest rates was proposed in 1980. Since then it has been the objective of many even recent studies and extensions. However, in spite of many deep theoretical studies, there are many errors, imprecisions or trivialities published in the financial literature. The main goal of this article is to correct some of them, and to show how relevant problems concerning CIR can be solved by easy applications of exponential martingales.

Usually it is assumed that risk premia are proportional to $\sqrt{r(t)}$; and linear risk premia are considered inadmissible; cf. Rogers (1995).

However, if one wants to work with the CIR model in Risk Neutral World (RNW) - the only world that can be observed for interest rates (IR) alone - then it turns out (in some cases) that linear risk premia are allowed. In this case the IR in the physical (real) world follow a different model. We solve this problem in section 1 using elementary application of the Girsanov change of measure.

The CIR model is related to the double square root Longstaff model. The wrong solution of pricing bonds with erroneous use of the Feynman-Kac formula was presented by Longstaff (1989) and a simple version was solved by Beaglehole \& Tenney (1992). The Longstaff "result" is still quoted in many even recent textbooks. We will show how to solve the latter case by an elementary use of exponential martingales.

2000 Mathematics Subject Classification: 91B28, 60G46, 60H30.

Key words and phrases: square root processes, exponential martingales, Girsanov theorem, risk premia.

The paper is in final form and no version of it will be published elsewhere. 
The Longstaff problem is similar to pricing default bonds in Merton's structural approach with CIR as a short rate.

We assume that asset prices follow a geometric Brownian motion and both models are driven by the same Brownian motion. The case of independent asset prices and IR was "solved" (in terms of the corresponding Laplace transform) by Wang (1999).

However in the intensity based approach with intensity correlated with IR in many cases we can obtain explicit results.

In section 4 we will comment how to model negative correlations (observed in some financial markets) in the CIR setting. We cannot agree with the statement by Dai \& Singleton (2000) that square-root diffusions are theoretically incapable of generating negative correlations.

An easy use of exponential martingales to price bonds in extended CIR was introduced in Szatzschneider (2002) and part of the study was reproduced in Musiela and Rutkowski (2004).

1. Linear risk premia. We stress that everything we can say about interest rates is deduced from prices of bonds or other interest rate derivatives, and these are priced in so called Risk Neutral World (RNW). In other words, dealing only with interest rates the RW (Real World) cannot be observed. Therefore in this case the concept of risk premium is dim.

If one wants to consider the RW for interest rates, this RW must be taken from assets.

We proceed with the construction of the RW for IR (interest rates) such that in the RNW the IR follow the CIR model.

For the CIR model in RW, linear risk premia are inadmissible; cf. Cox et al. (1985), Rogers (1995).

We will clarify what can be done and what cannot in a one dimensional financial market driven by Brownian motion, and asset prices that in the RW (under the law $P$ ) follow a geometric Brownian motion:

$$
d S(t)=S(t)[\sigma d W(t)+\mu d t] .
$$

Set (discounted prices) $Z_{t}=S_{t} / \beta_{t}$, where

$$
\beta_{t}=\exp \left(\int_{0}^{t} r(s) d s\right),
$$

and $r(s)$ is the spot IR in the RW. Now,

$$
d Z(t)=Z(t)[\sigma d W(t)+(\mu-r(t)) d t] .
$$

The RNW is defined as the probability law $Q(Q \sim P), t \leq T$ such that under $Q$

$$
d Z(t)=\sigma Z(t) d W^{*}(t)
$$

$W^{*}$ being another Brownian motion. It can be shown that if $r(t)$ is CIR (in real world) then such a $Q$ does not exist. An easy argument is based on explosion until $T=1$ of the process defined by

$$
d X(t)=d W(t)+X^{2}(t) d t,
$$

cf. Revuz \& Yor (1999) p. 384. 
But what we really want is CIR in the RNW. We prove the following:

THEOREM 1. If under $P$

$$
d r(t)=2 \widetilde{\sigma} \sqrt{r(t)} d W(t)+\left[\delta+2 \frac{\mu}{\sigma} \widetilde{\sigma} \sqrt{r(t)}-\left(2 \beta r(t)+\frac{2 \widetilde{\sigma}}{\sigma} r^{\frac{3}{2}}(t)\right)\right] d t,
$$

then for any $T>0$, there exists $Q \sim P$, for the process considered until time $T$ such that under $Q$ the interest rates follow

$$
d r(t)=2 \widetilde{\sigma} \sqrt{r(t)} d W^{*}(t)+(\delta-2 \beta r(t)) d t, \quad \widetilde{\sigma}, \delta, \beta>0 .
$$

Proof. Set $\widetilde{\sigma}=1=\sigma$. Because, by elementary calculations, the law $Q=Q^{\beta}$ is equivalent to the law $Q^{0}$ of the corresponding $\operatorname{BESQ}^{\delta}$ process $(\beta=0)$, and similarly $P=P^{\beta} \sim P^{0}$, it is sufficient to prove the equivalence of $P^{0}$ and $Q^{0}$. To stress that $\beta=0$, we change $r$ to $X$.

Applying Itô-Tanaka to $f(x)=x^{\frac{3}{2}}$ and occupation time formulas together with the fact that for $\mathrm{BESQ}^{\delta}$, the local time $L_{t}^{a}$ is 0 for $a \leq 0$ and $\delta>0$, we have that under $Q^{0}$ the exponential local martingale

$$
\begin{aligned}
\exp \left\{-\int_{0}^{t} X(s) d W^{*}(s)-\frac{1}{2} \int_{0}^{t} X^{2}(s) d s\right\} \\
=\exp \left\{-\frac{X_{t}^{\frac{3}{2}}}{3}+\frac{X_{0}^{\frac{3}{2}}}{3}+\frac{1}{2}(\delta+1) \int_{0}^{t} \sqrt{X(s)} d s-\frac{1}{2} \int_{0}^{t} X^{2}(s) d s\right\}
\end{aligned}
$$

is bounded by a constant $k(T)$.

Now it is easy to see that

$$
\eta_{t}=\mathcal{E}\left[\int_{0}^{\cdot}(X(s)-\mu) d W(s)\right]_{t}
$$

is a true martingale.

Moreover $\eta_{t}>0, P^{0}$ almost everywhere. We conclude that $Q^{0} \sim P^{0}$, and $Q \sim P$ on $\mathcal{F}_{T}$. A similar proof works if in the RW

$$
d S(t)=S(t)\{[(\lambda+1) r(t)+\mu] d t+\sigma d W(t)\} \quad \text { for any } \lambda<0 .
$$

Namely, there exists the corresponding model in RW such that in the RNW the IR follow the CIR model. We have just proved that in some cases the linear risk premia for the CIR model are admissible, of course in our formulation of the problem.

2. Longstaff model. In (1989) Longstaff proposed the so-called double square root model for instantaneous interest rates defined in Risk Neutral World by:

$$
d r(t)=2 \sqrt{r(t)} d W(t)+(1-\kappa \sqrt{r(t)}-2 \lambda r(t)) d t, \quad \kappa, \lambda>0 .
$$

In this study, for the sake of simplicity, we set $\sigma=1$ in the original model $\widetilde{r}(t)=\sigma r(t)$. Clearly:

$$
\begin{aligned}
r(t) & =y^{2}(t), \text { where } \\
d y(t) & =d W^{*}(t)-\left(\lambda y(t)+\frac{\kappa}{2} \operatorname{sgn} y(t)\right) d t .
\end{aligned}
$$


In 1992 Beaglehole \& Tenney showed that Longstaff's wrong formula for bond prices in his model gives the correct bond prices in the case of:

$$
r_{1}(t)=y_{1}^{2}(t) \quad \text { and } \quad d y_{1}(t)=d W(t)-\left(\lambda y_{1}(t)+\frac{\kappa}{2}\right) d t
$$

Longstaff in his study uses the Feynman-Kac approach and obtains the formula for bond prices of the form:

$$
P(t, T)=\exp \{m(t, T)-n(t, T) x-p(t, T) \sqrt{x}\}
$$

for some functions $m, n, p$, and $x=r_{t}$. However to apply the Feynman-Kac representation, $P(t, T)$ should be of class $C^{2}$ with respect to $x$, Karatzas \& Shreve (1991) theorem 7.6.

Some relaxation of this assumption is imaginable, but there is no possibility to make adjustments that could work for $P(t, T)$. The problem is of course at zero.

THEOREM 2. Longstaff's bond prices are correct if we replace $r(s)$ by $r_{1}(s)$.

Note. As we mentioned, this theorem has already been proved by Beaglehole \& Tenney. Our proof is an easy application of exponential martingales.

Proof. We have to calculate

$$
E_{x}\left(e^{-\int_{0}^{t} r_{1}(s) d s}\right)
$$

Let $f(s)$ and $g(s)$ be differentiable functions; then, clearly, for any $t$

$$
E\left[e^{\int_{0}^{t} f(s) W(s)+g(s) d W(s)-\frac{1}{2} \int_{0}^{t}(f(s) W(s)+g(s))^{2} d s}\right]=1 .
$$

The notation $E_{x}$ means that the process starts at $x$. In the sequel we shall use the notation $\propto$ for equality up to a deterministic factor. This factor is easy to calculate but we will not reproduce it here. Also for simplicity we choose $x=0$ in (3). The general case is analogous.

By the Girsanov theorem:

$$
\begin{aligned}
(2) & \propto E\left(e^{-\left(\frac{\lambda^{2}}{2}+1\right) \int_{0}^{t} W^{2}(s) d s-\frac{\lambda \kappa}{2} \int_{0}^{t} W(s) d s-\frac{\lambda}{2} W^{2}(t)-\frac{\kappa}{2} W(t)}\right) \\
& \propto E\left(e^{\int_{0}^{t}(f(s) W(s)+g(s)) d W(s)-\frac{1}{2} \int_{0}^{t}(f(s) W(s)+g(s))^{2} d s}\right)
\end{aligned}
$$

if and only if in $(0, t)$

$$
\begin{aligned}
f^{\prime}(s)+f^{2}(s) & =\lambda^{2}+2, \\
g(s) f(s)+g^{\prime}(s) & =\frac{\lambda \kappa}{2}, \\
f(t) & =-\lambda, \\
g(t) & =-\frac{\kappa}{2} .
\end{aligned}
$$

Therefore the problem of bond pricing in the Beaglehole \& Tenney model is reduced to entirely elementary calculations giving Longstaff's result which we do not reproduce here, cf. Longstaff's article.

This matching procedure does not work in the original Longstaff model, i.e. for calculations of

$$
P(0, t)=E_{x}\left(e^{-\int_{0}^{t} r(s) d s}\right) .
$$


An application of the Girsanov theorem leads to

$$
P(0, t) \propto E_{x}\left[e^{-\left(\frac{\lambda^{2}}{2}+1\right) \int_{0}^{t} W^{2}(s) d s-\frac{\kappa \lambda}{2} \int_{0}^{t}|W(s)| d s-\frac{\lambda}{2} W^{2}(t)-\frac{\kappa}{2}\left(|W(t)|-L_{t}^{0}\right)}\right] .
$$

In Szatzschneider (2004) we have shown how to calculate $P(0, T)$ where $T$ is an exponential random variable independent of the process. The main techniques were the Ray-Knight theorem or excursions, in the case $r(0)=0$. If one wants to solve the problem reducing first the process to zero, one should know the density of the hitting time of $y$ for the Ornstein-Uhlenbeck process starting at $x$. This problem does not have an explicit solution, cf. Göing, Yor (2003a), (2003b).

3. CIR and intensity based approach. Our goal is to calculate

$$
E_{x}\left(e^{-\int_{0}^{t}(r(s)+\lambda(s)) d s}\right)
$$

for $r(s)$ and $\lambda(s)$ dependent CIR processes, cf. Rutkowski, Bielecki (2001) p. 231 for details.

Assume that one can observe correlations between $r(s)$ (default free rate) and $\lambda(s)$ (the intensity of default, defined as $f(s) /(1-F(s))$ where $\left.F(s)=P(\tau \leq s), f(s)=F^{\prime}(s)\right)$. We will use very special dependence structure between $r(s)$ and $\lambda(s)$ that can generate the correlation one, and this structure will produce explicit formulas.

TheOREM 3. Assume that

$$
\operatorname{Cov}(r(t), \lambda(t))=\sum_{i=1}^{n} A_{i}\left(e^{-\kappa_{i} s}-e^{-2 \kappa_{i} s}\right)+B_{i}\left(1-e^{-\kappa_{i} s}\right)^{2} \quad A_{i}, B_{i}, \kappa_{i}>0
$$

In this case, using standard valuation of bonds in CIR setting (cf. Musiela \& Rutkowski 2004) the default bond prices are explicit.

Proof. For simplicity, set $n=1$. Our modelling is as follows. Set

$$
\begin{aligned}
& r(t)=r_{1}(t) \oplus r_{2}(t), \\
& \lambda(t)=\lambda_{1}(t) \oplus \lambda_{2}(t),
\end{aligned}
$$

with independent $r_{1}$ and $r_{2}, \lambda_{1}$ and $\lambda_{2}$. Also $r_{1}$ is independent of $\lambda_{1}$. Now set $\lambda_{2}(t)=$ $\epsilon r_{2}(t)$ for some $\epsilon>0$. Then

$$
d r_{2}(t)=2 \sigma \sqrt{r_{2}(t)} d W_{1}(t)+\kappa\left(\theta_{2}-r_{2}(t)\right) d t
$$

consequently

$$
d \lambda_{2}(t)=2 \sigma \sqrt{\epsilon} \sqrt{\lambda_{2}(t)} d W_{1}(t)+\kappa\left(\theta_{2} \epsilon-\lambda_{2}(t)\right) d t .
$$

With the use of the Pythagoras theorem define:

$$
\begin{aligned}
& \lambda(t)=2 \sigma \sqrt{\epsilon} \sqrt{\lambda(t)} d W_{2}(t)+\kappa(\theta-\lambda(t)) d t, \\
& r(t)=2 \sigma \sqrt{r(t)} d W_{3}(t)+\kappa(\widetilde{\theta}-r(t)) d t,
\end{aligned}
$$

with the restriction that:

$$
\theta>\epsilon \theta_{2}, \quad \tilde{\theta}>\theta_{2}
$$

In our setting

$$
\operatorname{Cov}(\lambda(t), r(t))=\epsilon \operatorname{Var}\left(r_{2}(t)\right) .
$$


Using the well known formula for the variance of the CIR we can write:

$$
\operatorname{Cov}(r(t), \lambda(t))=A\left(e^{-\kappa s}-e^{-2 \kappa s}\right)+B\left(1-e^{-\kappa s}\right)^{2}
$$

for some positive constants $A$ and $B$. Now $r(t)+\lambda(t)=r_{1} \oplus \lambda_{1}(t) \oplus(1+\epsilon) r_{2}(t)$ is the sum of independent standard CIR models. We believe that this method is the most explicit one to calculate prices of defaultable bonds in CIR setting.

4. Negative correlations. In this section we will explain briefly how to obtain negative correlations between factors in CIR framework.

The problem is extremely important and could lead toward future applications. In this first approach we will consider only two factors. As mentioned in Dai \& Singleton (2000), "The data on U.S. interest rates seems to call for negative correlations among the risk factors. Because CSR (Correlated Square Root) models are theoretically incapable of generating negative correlations, we conclude that they are not consistent with the historical behaviour of U.S. interest rates". Of course CIR models driven by the same Brownian motion cannot be negatively correlated. Therefore we have to relax the assumption "driven by the same Brownian motion".

A natural form to obtain a negative correlation could be by setting:

$$
\begin{aligned}
& r_{1}(t) \rightarrow \text { CIR driven by } W(t), \\
& r_{2}(t) \rightarrow \text { CIR driven by }-W(t) .
\end{aligned}
$$

The problem is that there is no easy approach to calculate

$$
E\left(\exp -\int_{0}^{t}\left(r_{1}(s)+r_{2}(s)\right) d s\right) \text {. }
$$

A similar comment was made by Schönbucher (2003), p. 175. Further we read "Alternatively one could restrict the specification to a squared Gaussian model". Our construction below represents the easiest and most explicit way to generate negative correlation.

THEOREM 4. Elementary valuation of prices of bonds can be performed if

$$
\operatorname{Cov}\left(r_{1}(t), r_{2}(t)\right)=2 \alpha \beta t(t-2 A B),
$$

which clearly can take negative values.

Proof. We restrict our attention, for simplicity, to two factors. Set

$$
\begin{aligned}
& r_{1}(t)=\hat{r}_{1}(t) \oplus \alpha(W(t)+A)^{2}, \\
& r_{2}(t)=\hat{r}_{2}(t) \oplus \beta(W(t)-B)^{2},
\end{aligned}
$$

for $\alpha, \beta>0$. Here $\hat{r}_{1}(t), \hat{r}_{2}(t)$ are CIR models, and $\hat{r}_{1}, \hat{r}_{2}, W$ are independent processes $(W(t)$ is Brownian motion).

Note that $(W(t)+A)^{2}$ and $(W(t)-B)^{2}$ are one dimensional squared Bessel processes driven by

$$
\begin{aligned}
& B_{1}(t)=\int_{0}^{t} \operatorname{sgn}(W(s)+A) d W(s), \\
& B_{2}(t)=\int_{0}^{t} \operatorname{sgn}(W(s)-B) d W(s) .
\end{aligned}
$$


$E\left(\exp -\int_{0}^{t} r_{i}(s) d s\right)$ for $i=1,2$ and $E\left(\exp -\int_{0}^{t}\left(r_{1}(s)+r_{2}(s)\right) d s\right.$ can be calculated once again by the standard martingale method.

Finally, one could be tempted to set:

$$
\begin{aligned}
& r_{1}(s)=\hat{r}_{1}(s)+\alpha W^{2}(s) 1(W(s)>0), \\
& r_{2}(s)=\hat{r}_{2}(s)+\alpha W^{2}(s) 1(W(s)<0) .
\end{aligned}
$$

However, calculations of $E \exp -\int_{0}^{t} r_{1}(s) d s$ are similar to pricing bonds in the Longstaff model, as explained in Pitman \& Yor (1982).

\section{References}

D. Beaglehole and M. Tenney (1992), Corrections and additions to "A nonlinear equilibrium model of the term structure of interest rates", Journal of Financial Economics 32, 345-353.

J. C. Cox, J. E. Ingersoll and S. A. Ross (1985), A theory of term structure of interest rates, Econometrica 53, 385-408.

Q. Dai and K. Singleton (2000), Specification analysis of affine term structure models, Journal of Finance 55, 1943-1978.

A. Göing-Jaeschke and M. Yor (2003a), A clarification note about hitting time densities for Ornstein-Uhlenbeck processes, Finance Stochast. 7, 413-415.

A. Göing-Jaeschke and M. Yor (2003b), A survey and some generalizations of Bessel processes, Bernoulli 9, 313-349.

I. Karatzas and S. E. Shreve (1991), Brownian Motion and Stochastic Calculus, 2nd ed., Springer.

F. Longstaff (1989), A nonlinear general equilibrium model of the term structure of interest rates, Journal of Financial Economics 23, 195-224.

M. Musiela and M. Rutkowski (2004), Martingale Methods in Financial Modelling, 2nd ed., Springer.

A. Revuz and M. Yor (1999), Continuous Martingales and Brownian Motion, 3rd ed., Springer.

L. C. G. Rogers (1995), Which model for term-structure of interest rates should one use?, in: Mathematical Finance IMA Vol. Math. Appl. 65, Springer, 93-115.

M. Rutkowski and T. Bielecki (2001), Credit Risk: Modeling, Valuation and Hedging, Springer.

P. Schönbucher (2003), Credit derivatives pricing models: models, pricing and implementation, Wiley, p. 175.

W. Szatzschneider (2004), Comments about CIR model, http://www.stat.ucl.ac.be/Samos2004/.

W. Szatzschneider (2002), The Cox, Ingersoll and Ross extended model, Revista Mexicana de Economía y Finanzas 1, 319-332.

D. F. Wang (1999), Pricing defaultable debt: some exact results, IJTAF 1, 95-99.

M. Yor (1992), Some aspects of Brownian Motion. Part I: Some Special Functionals, Birkhäuser. 
